\title{
A Study on Radar Target Detection using Space Time Adaptive Processing Algorithm and LCMV Algorithm
}

\author{
Kwan-HyeongLee ${ }^{1}$ \\ ${ }^{1}$ Division of Human IT Convergence, DaejinUniv.,POCHEON, Republic of Korea khlee@ daejin.ac.kr
}

Article History: Received: 11 January 2021; Accepted: 27 February 2021; Published online: 5 April 2021

\begin{abstract}
In this paper, we study the directionof arrival estimation of the desired target in adaptive array MV algorithm to update the weight, and the optimized weight removes the interference signal. The target signal is estimated using the optimized weight vector and the high resolution the direction of arrival estimation MUSIC algorithm. We calculate the inverse of the correlation matrix using the QR method to reduce the processing power consumption of the optimized weight. The optimal weight vector is applied to the proposed algorithm to estimate the desired target direction from the output power spectrum. The performance of the proposed method is compared with the existing method by simulation. The experimental method estimates three targets from the antenna received signal. The existing method did not estimate the three desired targets at $\left[-30^{\circ},-20,-10^{\circ}\right]$. The proposed method accurately estimates the desired three targets at $\left[-30^{\circ},-20,-10^{\circ}\right]$. In the $\left[-10^{\circ}, 0,10^{\circ}\right]$ target estimation, the existing method reduces the estimated resolution of the target, but the proposed method accurately estimates the target. We proved that the proposed method in the simulation was superior to the existing method.
\end{abstract}

Keywords:Direction of arrival, estimation, covariance, correlation matrix, weight.

\section{Introduction}

Signal processing technology has been studied to remove interference and noise signals in order to detect the desired signal from the signal received by the antenna system [1-2]. It is essential to remove interference and noise signals in order to detect the desired signal in wireless communication system. The existing interference signal removal method have been studied to reduce the power of interference signals with the antenna design changes and signal processing filtering technology. Information signal detection is called signal processing technology. This technology has been studied in radar, sonar, and mobile communication fields, and research to detect objects in a specific direction is called a target estimation method [3-[4]. One of the target estimation methods can detect the desired target using the arrival direction estimation technique. The target estimation method is a technique of radiating radio waves and detecting a desired signal from a signal received by a receiving antenna [5]. The target estimation method is a technique of removing interference and noise a received signal using a direction of arrival estimation algorithm. The direction of arrival estimation algorithm is a method of accurately estimating a desired information signal by minimizing a desired signal extraction error and considering characteristics of the signal.

The adaptive array antenna system has an advantage of eliminating interference signals, minimizing noise power, and avoiding frequency dependent phase distortion on the spread width signal [6-14]. However, broadband requires temporary filtering of array signal processing to suppress interference signals. The adaptive array system can use the beam-steering and beam-forming processing methods together to eliminate interference and accurately detect the desired signals [7-15]. The beam-steering method uses the desired signal direction as a complex weight at each tap output of the time-space processing algorithm. This method should be informed in advance of the location and direction of the receiving system. The beam directed method is full correlation technique that does not require reference signal detection in order to calculate weights by weighting the phase and gain for each tap of each antenna array element. The beam-forming is called post-correlation method as a method of detecting a signal using the adaptive algorithm to calculate the weight and maximizing the signal-tonose ratio. The adaptive array system also improves the processing gain of the receivers. It maximized the method of having signal characteristics of expansion / non-expansion by reducing the unexpanded signal bandwidth to a minimum. Designing the bandwidth of the receiver's code/carrier tracking loop in a narrow band improves interference removal.However, reducing the bandwidth of code/carrier tracking loops slows down the response speed, which reduces the tracking loop performance and reduces the ability to eliminate signal interception. Conversely, expanding bandwidth makes it difficult to effectively block outside noise [8].

In this stud, we use space time processing algorithm and Linear Constraint Minimum Variance (LCMV) algorithm in adaptive array antenna system to detect the desired signal. This research method applies the LCMV 
algorithm to the adaptive array antenna system to complement characteristics sensitive to differences in RF characteristics between channels and remove the interference and noise signals to detect the desired target signal. The composition of this paper is as follow. Section 2 describes the signal analysis of the LCMV algorithm, and Section 3 proposes a LCMV algorithm of matrix inversion. Section 4 analysis the performance of the proposed algorithm, and closes the paper in section 5 .

\section{Spatial Adaptive Processing}

The LCMV algorithm in as minimizes the power of signal received from the adaptive array antenna. It is an effective signal suppression method for strong interference signals because a smaller gain is set in the direction in which the interference signal is present. However, the LCMV algorithm poses a risk factor that will suppress the desired signal because it does not use any information compared to other space time adaptive processing (STAP) algorithms.This algorithm should set constraints on gains in the desired signal direction to prevent suppression of these desired signals. In addition, the LCMV algorithm is basically developed on the premise that each of the delay characteristics from each antenna element through RF and IF circuits to digital circuits are exactly matched, and this is sensitive to these characteristics [9].When the number of arrays in the antenna is $\mathrm{K}$ and the tab delay is $\mathrm{P}$, the receiving signal vector can be expressed as follows:

$$
\mathrm{x}(\mathrm{t})=\left[\mathrm{x}_{1}(\mathrm{t}), \mathrm{x}_{2}(\mathrm{t}), \cdots, \mathrm{x}_{\mathrm{k}}(\mathrm{t})\right]^{\mathrm{T}}
$$

The output signal of the adaptive array antenna is expressed as follows by the antenna reception signal and weight [10].

$$
\mathrm{Y}(\mathrm{t})=\sum_{\mathrm{k}=1}^{\mathrm{K}} \sum_{\mathrm{j}=0}^{\mathrm{P}-1} \mathrm{x}_{\mathrm{k}}(\mathrm{t}-\mathrm{j} \mathrm{T}) \mathrm{w}_{\mathrm{k}, \mathrm{j}}=\mathrm{W}^{\mathrm{H}}(\mathrm{t}) \mathrm{x}(\mathrm{t})
$$

Here, ()$^{H}$ is the hermit matrix, and the weight vector $\mathrm{W}$ is expressed as follows:

$$
\mathrm{W}(\mathrm{t})=\left[\mathrm{w}(\mathrm{t}), \mathrm{w}_{2}(\mathrm{t}), \cdots, \mathrm{w}_{\mathrm{k}}(\mathrm{t})\right]^{\mathrm{T}}
$$

The expectation of the output power signal of the adaptive array antenna can be expressed as follows:

$$
\begin{aligned}
E\left[|\mathrm{Y}(\mathrm{t})|^{2}\right] & =\mathrm{E}\left[\left(\mathrm{W}^{\mathrm{H}}(\mathrm{t}) \mathrm{x}(\mathrm{t})\right)\left(\mathrm{W}^{\mathrm{H}}(\mathrm{t}) \mathrm{x}(\mathrm{t})\right)^{\mathrm{H}}\right] \\
& =\mathrm{W}^{\mathrm{H}} \mathrm{E}\left[\mathrm{x}(\mathrm{t}) \mathrm{x}(\mathrm{t})^{\mathrm{H}}\right] \mathrm{W} \\
& =\mathrm{W}^{\mathrm{H}}(\mathrm{t}) \mathrm{C}(\mathrm{t}) \mathrm{W}(\mathrm{t})
\end{aligned}
$$

Here, $C(t)=E\left[x(t) x(t)^{H}\right]$ is the array correlation matrix including spatial correlation of signals received from the antenna array element. The array signal should be optimized to fit the weight vector to the desired gain constraint in order to minimize the output power signal [11]. The gain constraint is as follows:

$$
\mathrm{W}^{\mathrm{H}}(\mathrm{t}) \mathrm{D}=\mathrm{g}
$$

Here, $\mathrm{D}=\left[s_{1}, s_{2}, \cdots, s_{L}\right]$ is a steering matrix. This steering matrix is a matrix containing $\mathrm{L}$ steering vector for which $\mathrm{L}$ gain constraints are set. The vector $\mathrm{G}$ means fixed gain in the constraint direction, and each $\mathrm{L}$ vectors in the steering matrix form one constraint. The method of setting constraint for beamforming is divided into single LCMV beam-former, multiple LCMV beam-former, and unconstrained power minimization [12-13]. The signal LCMV beam-former constraint is as follows:

$$
\mathrm{W}^{\mathrm{H}}(\mathrm{t}) \mathrm{d}=\mathrm{g}
$$

Here, $d$ represents one steering vector for single beamforming. This condition applies the same weight vector for all signals source directions. The multiple LCMV beam-former constraint is as follows:

$$
W^{\mathrm{H}}(\mathrm{t}) \mathrm{D}=\mathrm{g}
$$

Here, D represents multiple steering vector for multiple beamforming. This condition applies different weight vector for all signal source directions. The unconstrained power minimization LCMV beam-former constraint is as follows:

$$
W^{H}(t) d=1
$$

In equation (10), $d=[1,0 \cdots, 0]^{T}$ must have a weight of 1 for the reference element of the antenna. $\mathrm{N}$ nulls can be used for suppression an interference signal by using one degree of freedom as a constraint. The unconstrained power minimization operates only in a direction that minimizes the input signal, so the weighted vector $w=0$. Considering the weighted vector, the unconstrained power minimization constraint is set at $g=1$. The unconstrained power minimization constraint greatly increases the risk of suppressing the desired signal, but decreases the likelihood that the desired signal will be suppressed because the power of the information signal is smaller than the noise level. This paper applies the unconstrained power minimization constraint which can use $\mathrm{N}-1$ nulls. 


\section{Proposal LCMV Algorithm of Matrix Inversion}

The LCMV algorithm is divided into spatial-nulling algorithm and STAP algorithm according to covariance matrix. Obtain the weight of the inverse matrix from the covariance matrix. It is divided into inverse matrix algorithm and QR algorithm that performs a direct inverse sequence according to the method of calculation of the inverse matrix. The interference and noise signals are removed by obtaining the optimal weight of each algorithm. Figure 1 shows block diagram of the LCMV algorithm. The LCMV algorithm is applied by converting the intermediate frequency signal to digital signal (ADC). At this time, LCMV algorithm calculates the covariance matrix using spatial nailing and STAP to obtain the optimal weight. The optimal weight acquisition method calculates the covariance matrix using the correlation matrix and uses the inverse of the covariance matrix to obtain the optimal weight.

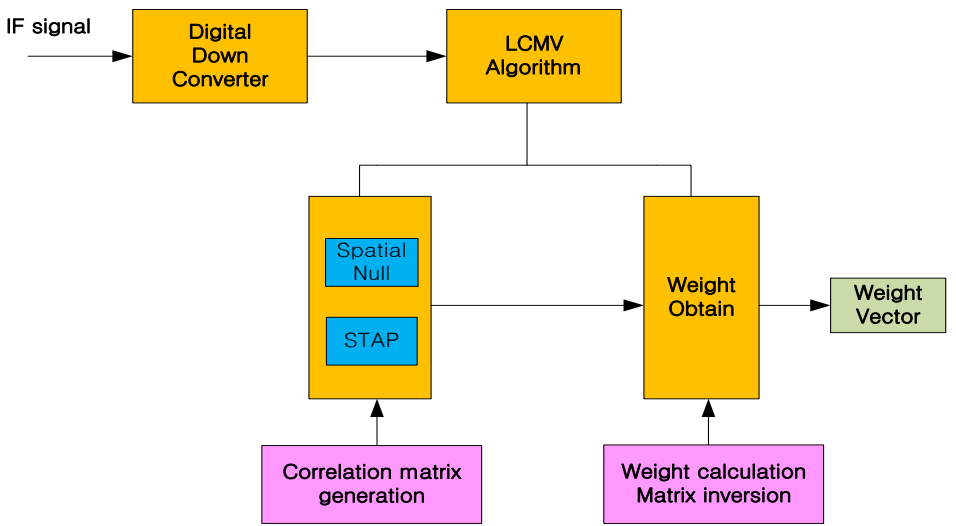

Figure 1. Spatial Adaptive Processing System.

This study uses the unconstrained power minimization constraint method for beamforming. When the unconstrained power minimization constraint is used to minimize the output signal of the adaptive array antenna, the output signal can be expressed as follows using Lagrange multiplier.

$$
\begin{gathered}
\nabla_{\mathrm{w}}\left[\mathrm{E}\left[|\mathrm{Y}(\mathrm{t})|^{2}\right]+\mathrm{g}\right]=\nabla_{\mathrm{w}}\left(\mathrm{W}^{\mathrm{H}}(\mathrm{t}) \mathrm{C}(\mathrm{t}) \mathrm{W}(\mathrm{t})+\lambda \mathrm{W}^{\mathrm{H}}(\mathrm{t}) \mathrm{d}\right) \\
\mathrm{C}(\mathrm{t}) \mathrm{W}(\mathrm{t})+\lambda \mathrm{d}=0
\end{gathered}
$$

When the weight vector is obtained for the smallest point of equation (11), the optimized weight vector is as follows:

$$
\begin{gathered}
\mathrm{W}_{\text {op }}(\mathrm{t})=-\lambda \mathrm{C}^{-1}(\mathrm{t}) \\
\mathrm{d}^{\mathrm{H}} \mathrm{W}_{\mathrm{op}}(\mathrm{t})=-\lambda \mathrm{d}^{\mathrm{H}} \mathrm{C}^{-1}(\mathrm{t})=\mathrm{g} \\
-\lambda=\frac{\mathrm{g}}{\mathrm{d}^{\mathrm{H}} \mathrm{C}^{-1}(\mathrm{t}) \mathrm{d}} \\
\mathrm{W}_{\mathrm{op}}(\mathrm{t})=\frac{\mathrm{g} \mathrm{C^{-1 } ( \mathrm { t } ) \mathrm { d }}}{\mathrm{d}^{\mathrm{H}} \mathrm{C}^{-1}(\mathrm{t}) \mathrm{d}}
\end{gathered}
$$

The optimal weight vector obtained in equation (16) has a lot of processing power due to the inverse of the vector. We apply $\mathrm{QR}$ algorithm for an efficient methods with low processing power consumption. The input signal vector of the antenna is separated into two matrices of $\mathrm{QR}$ decomposition.

$$
\mathrm{x}(\mathrm{t})=\mathrm{QR}
$$

Here $\mathrm{Q}$ and $\mathrm{R}$ are orthogonal matrix and upper triangular matrix, respectively. The input signal vector of the antenna from $\mathrm{Q}^{H} \mathrm{Q}=\mathrm{Q}^{H} \mathrm{Q}=1, \mathrm{Q}^{H}=\mathrm{Q}^{H-1}$ is as follows:

$$
\mathrm{X}^{-1}=\mathrm{R}^{-1} \mathrm{Q}^{-1}=\mathrm{R}^{-1} \mathrm{Q}^{H}
$$

We apply the modified Gram-Schmidt QR factorization to obtain the inverse of the upper triangulation of equation (17). Classical Gram-Schmidt QR factorization has the advantage of relatively low error in fixed precision calculation than the modified Gram-Schmidt QR factorization. The modified Gram-Schmidt algorithm is developed as pseudo code to find the inverse of the upper triangle. When the inverse of the upper triangle is calculated, the inverse of the antenna input signal is obtained by multiplying the Q matrix by the Hermitian transpose.

\section{Computer Simulation}

This chapteranalysis the performance of the methods proposed in this study with general methods through simulation.The existing general method is the MUSIC method, and the proposed method is applied the LCMV algorithm to the MUSIC method.The receiving system used an adaptive array linear antenna, and the array element spacing was set at half wavelengths to avoid the grading lobes.The signal-to-noise ratio was $20 \mathrm{~dB}$ and the array element was eight. Figure 2 and 3 estimate the target using a signal-to-noise ratio of $20 \mathrm{~dB}$ and 8 antenna array at $\left[-30^{\circ},-20^{\circ},-10^{\circ}\right]$.Figure 2 shows the three predicted results using only the MUISC method. 
Figure 3 did not accurately estimate the desired three targets. Three main lobes of the beam shall be represented in the desired target direction. However, it can be seen in Figure 2 that about one beam staple appeared. The desired target estimation accuracy shows good performance by improving resolution by increasing transmission power or number of antennas. However, it is not efficient in the system to increase the number of antenna array elements and increase the transmission power. Figure 3 shows a graph of the desired target estimates by the method proposed in this study. It can be seen that the beam main salts are accurate compared to Figure 2. Figures 4 and 5 show a graph of three desired targets at $\left[-10^{\circ}, 0^{\circ},-10^{\circ}\right]$ using 10 array elements. Figure 5 is the result of three predictions using only the MUISC method, which increases the number of antennas, resulting in better resolution than Figure 2.After increasing the number of antennas, three desired targets were estimated. That is, comparing Figure 4 with Figure 2, it can be seen that the resolution is improved by increasing the number of antennas. However, the three main lobes of the beam did not appear exactly at the desired target. Figure 5 is a graph that estimates desired target using the method proposed in this study. It accurately estimates three targets.

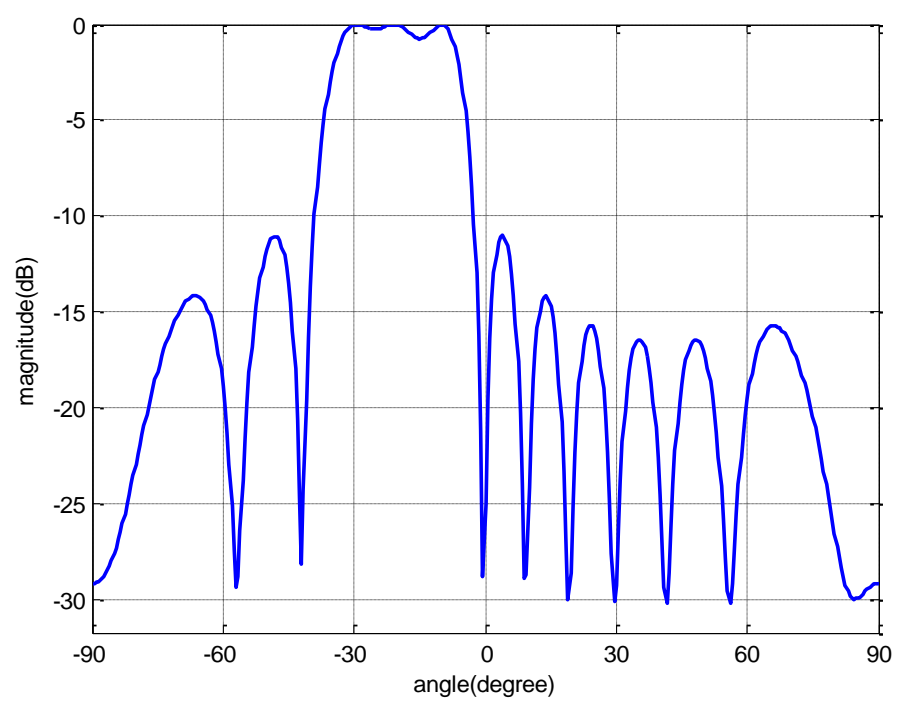

Figure 2. Existing MUSIC DoAat $\left[-30^{\circ},-20^{\circ},-10^{\circ}\right]$

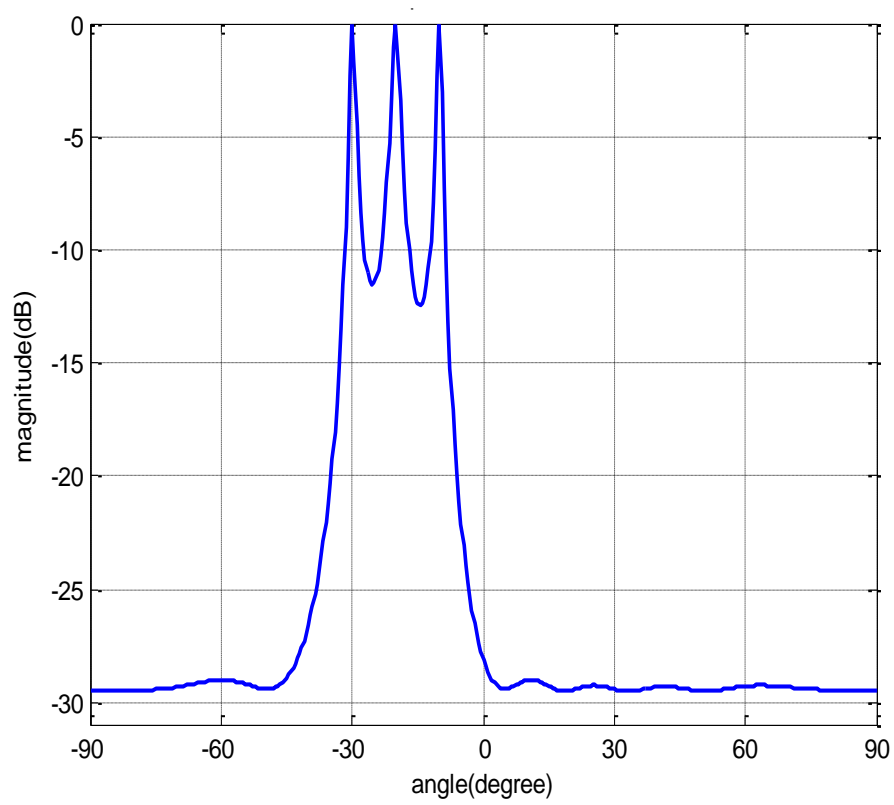

Figure 3. Proposed Algorithm DoAat $\left[-30^{\circ},-20^{\circ},-10^{\circ}\right]$ 


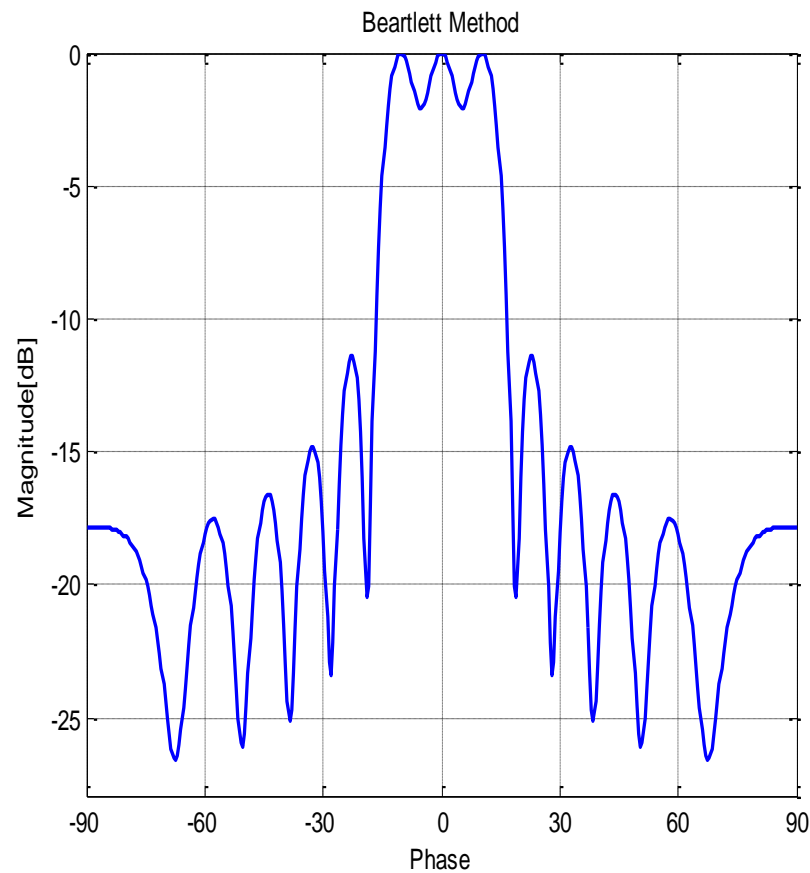

Figure 4. Existing MUSIC DoAat $\left[-10^{\circ}, 0^{\circ}, 10^{\circ}\right]$

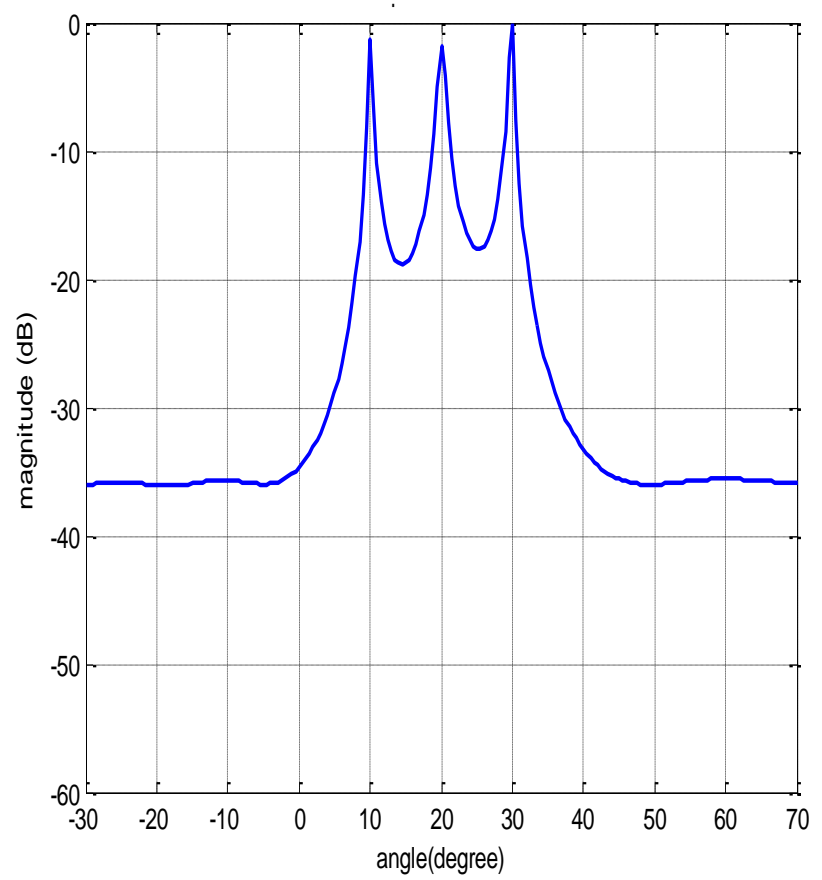

Figure 5. Proposed Algorithm DoAat $\left[-10^{\circ}, 0^{\circ}, 10^{\circ}\right]$

\section{Conclusion}

In this study, the STAP- LCMV algorithm was proposed to estimate the direction of arrival of the target when an interference waves existed in the radio channel. When an interference signal is received, the target signal is attenuated and an error occurs between the sample angle and the interference signal that forms the directional matrix, resulting in a significant degradation of the receiver's performance. This study estimated the direction of arrival of desired targets in space using optimal weight and QR inverse. The MUSIC method was used to estimate the direction of arrival. This is because the MUSIC method has better resolution than other conventional methods of estimating direction of arrival. The performance of the proposed method was compared with the existing MUSIC method to confirm. To increase resolution to less than 3 degrees using MUSIC method, the number of array antennas should increase or the signal-to-noise ratio should be high. The difference between the existing methods of estimating the direction of arrival is the superiority of the method proposed in this study in the accuracy of the estimation of the direction of arrival. The reason forthis was that the maximum signal-to- 
interference noise ratio was calculated at each moment to calculate the optimal weight. In this study, the direction of arrival was accurately estimated over the existing method and the resolution was also improved. In addition, the method proposed in this study was proven to be superior to the existing method when only correlation interference signals were present in radio channels.

\section{References}

1. Merrill, I.,\& McGraw, Hill. (2000). Introduction to Radar System.

2. Bassem, Mahaf, R., \&ChapmanHall (2000). Radar System Analysis and Design using Mathlab.

3. Sarma, T, C.; Rao, R. V. S.: Venugopal, C.; and kumar, P.V. (2004). Design of High Bit Rate Digital Correlator. IEEE TENCON Conference, 4(1).

4. Lukin, K. A.; Zenlyniy, O. V.; Tatyako, s.; and Pascazio, V. (2016). FPGA-base Time Integrating Multichannel Correlator for Noise Radar Applications. IEEE MSMW Conference, 1(1).

5. Daku. B. L. F; and Mclntyre. M. C. (1996). Short Time Correlator Delay Estimation in the Presence of Interfering Sources. IEEE ICASSP Conference, 1(1).

6. Dikmese, S.;Kavak, A.; Kucuk, K.; Sahin, S.; Tangel, A.; and Dince, H. (2010). Digital Signal Processor against Field Programmable Gate Array Implementations of Space Code Correlator Beamformer for Smart Antennas. IET Microwaves, antennas \&Propagation, 4(5), 593-599.

7. Hong, W. and Zhu. (1993). Radar Cross-Section of Dielectric-Coated Conduction Cylinder Loaded with Periodic Metallic Strips using Mixed SDM, CGM and FFT:TE Incidence. IET Microwaves, antennas and Propagation, 140(10), 373-377.

8. Sharef, Neemat.; Oleg, Kransnow.; and Alexander, Yarovoy. (2018). An Interference Mitigation Technique for FMCW Radar using Beat-Frequencies Interpolation in the STFT Domain. IEEE Transactions on Microwave Theory and Techniques,67(11), 1207-1220.

9. Seifallah, Jardar.; Sajid, Ahmed.; and Mohamed, Slim. Alouini. (2017). Low Complexity Moving Target Parameter Estimation for MIMO Radar using 2D-FFT, IEEE Transactions on Signal Processing, 65(6), 4745-4755.

10. Unnikrishna, Pillai. S. \& John Wiley. (1989). Array Signal Processing.

11. Xial, Chun.Nie.; Ning, Yuan.; Wei, Li.; and Yeow. Beng. Gan. (2008). Fast Analysis of RCS over a Frequency Band using Pre-Corrected FFT/AIM and Asymptotic Waveform Evaluation Technique. IEEE Transactions on Antennas and Propagation, 56(1), 3526-3533.

12. Tran, T. V.; and McCowen, A. (1993). An improved Pulse-Basis Conjugate Gradient FFT method for the thin conduction plate problem. IEEE Transactions on Antennas and Propagation, 41(2), 185-190.

13. Akopian, D. (2005). Fast FFT based GPS Satellite Acquisition Methods. IET Radar, Sonar and Navigation, 152(4), 277-286.

14. Afonso, A., \& Silva, J. (2019). Efectos de la políticamonetaria de la zona del euro en los sectoresinstitucionales: el caso de Portugal. Cuadernos de Economía, 42(120).

15. Armijos-Bravo, G. (2019). Inequidadessocioeconómicasen el uso de cuidadospaliativosen Europa. Cuadernos de Economía, 42(118). 